\title{
REVISED
}

\section{Comparison of Ribotyping and Sequence-Based Typing for Discriminating Among Isolates of Bordetella bronchiseptica}

\author{
Karen B. Register ${ }^{1}$, Tracy L. Nicholson ${ }^{2}$ and Brian W. Brunelle ${ }^{3}$ \\ ${ }^{1}$ Ruminant Diseases and Immunology Research Unit, ${ }^{2}$ Virus and Prion Research Unit or ${ }^{3}$ Food \\ Safety and Enteric Pathogens Research Unit, USDA/Agricultural Research Service/National \\ Animal Disease Center, 1920 Dayton Avenue, Ames, Iowa 50010
}

Running Title: genotyping B. bronchiseptica

*Send proofs and correspondence to:

Dr. Karen B. Register

Ruminant Diseases and Immunology Research Unit USDA/ARS/National Animal Disease Center 1920 Dayton Avenue

Ames, Iowa 50010

Phone: (515) 337-7700

Fax: (515) 337-7458

E-mail: karen.register@ars.usda.gov 


\begin{abstract}
$P v u I I$ ribotyping and MLST are each highly discriminatory methods for genotyping Bordetella bronchiseptica, but a direct comparison between these approaches has not been undertaken. The goal of this study was to directly compare the discriminatory power of $P v u I I$ ribotyping and MLST, using a single set of geographically and genetically diverse strains, and to determine whether subtyping based on repeat region sequences of the pertactin gene $(p r n)$ provides additional resolution. One hundred twenty-two isolates were analyzed, representing 11 mammalian or avian hosts, sourced from the United States, Europe, Israel and Australia. Thirtytwo ribotype patterns were identified; one isolate could not be typed. In comparison, all isolates were typeable by MLST and a total of 30 sequence types was identified. An analysis based on Simpson's Index of Diversity (SID) revealed that ribotyping and MLST are nearly equally discriminatory, with SIDs of 0.920 for ribotyping and 0.919 for MLST. Nonetheless, for ten ribotypes and eight MLST sequence types, the alternative method discriminates among isolates that otherwise type identically. Pairing prn repeat region typing with ribotyping yielded 54 genotypes and increased the SID to 0.954 . Repeat region typing combined with MLST resulted in 47 genotypes and an SID of 0.944. Given the technical and practical advantages of MLST over ribotyping, and the nominal difference in their SIDs, we conclude MLST is the preferred primary typing tool. We recommend the combination of MLST and prn repeat region typing as a high-resolution, objective and standardized approach valuable for investigating the population structure and epidemiology of $B$. bronchiseptica.
\end{abstract}

Key Words: Bordetella bronchiseptica; MLST; ribotyping; pertactin; pubMLST 


\section{Introduction}

Bordetella bronchiseptica is a widespread bacterial pathogen that infects a variety of domesticated and wildlife animal species. It causes tracheobronchitis (kennel cough) in dogs, bronchopneumonia in numerous laboratory, companion and wild animals, neonatal pneumonia in piglets and is an important contributor to swine atrophic rhinitis and porcine respiratory disease complex (Brockmeier et al. 2012). Human infections occasionally occur, most frequently in immunocompromised individuals, but related illness in healthy adults and children has also been reported (Lo Re et al. 2001; Llombart et al. 2006; Wernli et al. 2011).

Multilocus sequence typing (MLST), based on partial sequencing of seven housekeeping genes (Diavatopoulos et al. 2005), and PvuII ribotyping (Register et al. 1997; Register and Magyar 1999) have proven useful to distinguish among strains of B. bronchiseptica. Both methods are highly discriminatory and have been used to infer relationships among isolates and to identify likely sources of exposure. It is unclear which of these methods is most discriminatory since they have not been directly compared using a single set of isolates and relatively few isolates have been typed by more than one method. Comparison among isolates of the GGXXP and PQP repeat regions of the gene encoding the surface-exposed adhesin pertactin (prn) can also be an informative adjunct approach since polymorphisms occur at a relatively high frequency (Boursaux-Eude and Guiso 2000; Register 2001; Register 2004). The purpose of this study is to directly compare the discriminatory power of MLST and PvuII ribotyping and to ascertain whether prn repeat region sequencing provides an additional level of discrimination using a single set of geographically and genetically diverse strains.

\section{Materials and methods}

2.1 Bacterial isolates, growth conditions and DNA preparation 
A total of 122 isolates of $B$. bronchiseptica was analyzed, selected to represent diverse host and geographic origins. Included among these isolates are representatives of the $22 P v u I I$ ribotypes so far recognized (Register et al. 1997; Register and Magyar 1999; Register et al. 2000; Register et al. 2015a) and 25 of the 61 MLST sequence types (STs) currently associated with $B$. bronchiseptica isolates in the Bordetella PubMLST database

(http://pubmlst.org/bordetella). Isolates were sourced from the United States, Europe, Israel and Australia and include 24 from humans, 20 from seals, 17 from pigs, 13 from turkeys, 12 from rabbits, 10 from dogs, 8 from guinea pigs, 7 from horses, 6 from cats, 3 from koalas, 1 from a leopard and 1 of unknown origin. All additional information related to the provenance of the isolates can be found in the Bordetella Isolates MLST database (http://pubmlst.org/perl/bigsdb/bigsdb.pl?db=pubmlst_bordetella_mlst_isolates; Jolley and Maiden 2010). Corresponding database isolate ID numbers are 6, 7, 18-24, 27, 69, 71, 72, 75$78,80,82-85,87,92,93,101,104,105,108-110,113,118-120,122-128,145,326,358-370$, 379-382 and 444-504.

Bacteria were cultured at $37^{\circ} \mathrm{C}$ for 18 to $36 \mathrm{~h}$ on Bordet-Gengou agar supplemented with 10\% sterile, defibrinated sheep's blood. Chromosomal DNA was purified using a commercially available kit (Promega, Madison, WI) and quantified with PicoGreen reagent (Invitrogen, Carlsbad, CA).

\subsection{Ribotyping}

$P v u I I$ ribotyping was carried out as described (Register and Magyar 1999). Photographs of $P v u \mathrm{II}$ ribotyping gels were scanned and the fragment pattern of each isolate was compared using GelCompar II software (Applied Maths). Similarity between all possible pairs of fingerprint profiles using the coefficient of Dice (Sneath and Sokal 1973) was calculated by the cluster analysis module of the software. Dendrograms were derived from a matrix of similarity 
values by UPGMA (unweighted pair group method with arithmetic mean). Ribotype (RT) 1RT22 have been previously described (Register et al. 1997; Register and Magyar 1999; Register et al. 2000; Register et al. 2015a). Novel patterns were assigned to newly defined RTs. RTs for 78 of the isolates included in this study have been reported previously (Register et al. 1997; Register and Magyar 1999; Register et al. 2000; Register 2004; Rath et al. 2008; Register et al. 2015a).

\subsection{Sequence-based typing}

PCR amplicons used for sequencing were treated with ExoSAP-IT (USB Corporation) and sequenced directly by fluorescence-based cycle sequencing using Applied Biosystems Big Dye Terminator version 3.1 on an Applied Biosystems 3130 XL Genetic Analyzer at the National Animal Disease Center Genomics Unit. Vector NTI Advance was used for sequence editing and analysis. Final consensus sequences represent a minimum of three-fold coverage with at least one read from each strand.

The scheme of Diavatopoulos et al. (2005) was employed for MLST analysis. MLST allele sequences and profiles were evaluated using the PubMLST Bordetella Sequence and Profile Definitions Database, available via a link on the home page of the Bordetella PubMLST web site (http://pubmlst.org/bordetella/). For each isolate, amplicon sequences from the seven target genes were concatenated and used to construct a UPGMA tree utilizing the Maximum Composite Likelihood distance estimation model with 1000 bootstrap replicates. All positions containing gaps were eliminated from the dataset (complete deletion option). The final dataset includes a total of 2924 nucleotide positions. Phylogenetic analyses were conducted using MEGA6 version 6.05 (Tamura et al. 2013). STs for 61 isolates included in this study have been reported previously (Diavatopoulos et al.; 2005; Rath et al. 2008; Register et al. 2015a). Those 
for an additional 21 isolates were deduced from genome sequence data (Register et al. 2015b; GenBank accession \#NZ_JGWN01000000).

Pertactin gene repeat regions were amplified by PCR, sequenced and analyzed as described (Register 2004), using the nomenclature of Register (2001) to classify allelic variants. Briefly, the epithet 1-Nx, where $\mathrm{N}$ is an Arabic numeral indicating the number of GGXXP repeats and $\mathrm{x}$ is a lowercase letter used to distinguish additional unique insertions, substitutions, or deletions, is used to describe region 1 variants. Similarly, region 2 variants are categorized using the epithet 2-Nx, with $\mathrm{N}$ representing the number of PQP repeats. An additional Arabic numeral is used to discriminate between different DNA sequence alleles with conservative substitutions that result in identical predicted amino acid sequences. Pertactin repeat region sequences from 86 isolates included in this study were previously reported (Register 2001; Parkhill et al. 2003; Register 2004; Diavatopoulos et al. 2005; Rath et al. 2008; Register et al. 2015a) or are available from genome sequence data (Register et al. 2015b; GenBank accession \#NZ_JGWN01000000).

\subsection{Comparison of ribotyping and MLST}

The method of Hunter and Gaston (1988) was used to calculate Simpson's Index of Diversity (SID) for comparisons of the discriminatory power of $P v u I I$ ribotyping and MLST.

2.5 DNA sequences

The GenBank accession numbers for sequences newly reported here are KX547658KX548009.

\section{Results}

3.1 Ribotype analysis 
A total of $32 P v u I I$ ribotype patterns was identified from the 122 B. bronchiseptica isolates evaluated based on various combinations of 35 restriction fragments ranging in size from $\sim 1.8 \mathrm{~Kb}$ to $5.9 \mathrm{~Kb}$ (depicted graphically in Fig. 1). DNA from one isolate, cultured from a guinea pig in Australia, could not be digested completely with $P v u I I$ such that an RT could not be assigned. The ten RTs newly identified in this study, RT23-RT32, include both novel restriction fragments as well as novel combinations of previously known fragments.

Cluster analysis of the RT patterns delineates six major clusters (Fig. 2). Clusters I-V were defined previously on the basis of RT1-RT22 (Register et al. 2015a). In the current study, a novel, highly divergent cluster (VI) was identified consisting of RT29 and RT30, each represented by a single human isolate. Cluster VI has $<42 \%$ similarity to other clusters, which otherwise share a minimum of $\sim 62 \%$ similarity. The distribution of the original 22 RTs among clusters I-V was unaffected by the addition of the new RTs reported here but, with the exception of Cluster II, each cluster gained one or more of the newly defined RTs.

The distribution of isolates among RTs and clusters on the basis of the host of origin is detailed in Table 1. Clusters I-V each contain isolates from at least four different hosts while Cluster VI is so far comprised of only human isolates. Because of the limited number of Cluster VI isolates currently available it is unclear whether the cluster is truly host-restricted. Although no other cluster is restricted to a single host, all or a majority of isolates from several hosts are restricted to particular clusters and RTs. Specifically, those from pigs (16/17), turkeys (9/13), dogs (8/10) and cats (6/6) have RTs largely found in Cluster I while all isolates from seals $(n=20)$ are either RT19 or RT22, both within Cluster II. All koala isolates analyzed $(n=3)$ are RT6, in Cluster III, but additional, uniquely sourced isolates are needed to more thoroughly evaluate the strength of this association.

There is no obvious correlation between cluster and geographic origin. Isolates from the United States $(n=64)$ are found in all clusters and those from continental Europe are scattered 
among clusters I, II, IV and V. Isolates from the United Kingdom $(n=21)$ are found only in clusters I and II but this is largely due to the limited heterogeneity among isolates from seals, the host of origin for 15 of the isolates in the group, all RT19. The remaining RT19 isolate was recovered from a seal off the Danish coast. Of the six Australian isolates evaluated, one is nontypeable and the remainder segregate between two relatively divergent branches of the RTbased phylogeny, with two pig isolates in Cluster I (RT2 and RT3) and three koala isolates in Cluster III (RT6).

\subsection{MLST analysis}

Thirty STs were identified from the 122 B. bronchiseptica isolates evaluated, comprising seven major clades (Fig. 3). Table 2 details the distribution of isolates among clades and STs according to the host of origin. Three or more host species are represented within all clades having at least six isolates. However, isolates from two hosts are restricted to single clades; turkey isolates are found solely in Clade I, with 8/13 typed as ST28, while isolates from seals are either ST33 or ST11, both in Clade IV. Also found predominantly in Clade IV are isolates from pigs (15/17), rabbits (9/12), horses (6/7) and cats (4/6). Isolates cultured from other hosts are more broadly scattered among clades. An exclusive association between ST and host was noted only for ST11, which is comprised wholly of isolates from seals. There is additionally a strong correlation between ST28 and host of origin; with the exception of a single human isolate, all ST28 strains originate from turkeys.

No correlation between clade and geographic origin is evident. Isolates from the United States and continental Europe are found in all clades with six or more members. The two isolates in Clade III were both acquired from koalas in Australia. All but a single isolate from the United Kingdom are in clade IV but, as noted above, this is likely due to the predominance of 
seal isolates within that group rather than a geographic association per se. Isolates acquired from Australia segregate between two closely related lineages, Clade III $(n=2)$ and Clade IV $(n=4)$.

\subsection{Comparison between ribotyping and MLST}

The SIDs for ribotyping and MLST are nearly identical, 0.920 and 0.919 , respectively. These values indicate that two randomly isolated strains would be classified as distinct RTs or MLSTs $\sim 92 \%$ of the time. Both methods are sufficiently discriminatory for typing purposes based on the recommended threshold of $\geq 0.900$ (Hunter and Gaston 1988). However, for ten RTs and eight STs, the alternative method discriminates among isolates that otherwise type identically (Table 3). For six RTs (RT4, RT5, RT6, RT7, RT9 and RT10) and five STs (ST6, ST7, ST10, ST12, and ST33) the alternative method assigns the affected isolates to multiple clades or clusters, often comprising relatively distant branches of the corresponding phylogeny (Figs. 2 and 3; Table 3). For example, RT4 isolates are parsed among MLST clades IV, V and VI, RT5 and RT9 isolates are found in clades IV and VI, RT7 isolates segregate to clades IV and V while RT10 isolates have MLSTs representing clades I and VI. Conversely, ST7 isolates are split among RT clusters I, III, and V, ST10 isolates fall into clusters I, II and V, ST12 isolates are found in clusters I and V while ST33 isolates are represented in clusters II, III and V.

\subsection{Pertactin repeat region analysis}

The DNA sequences encoding the GGXXP and PQP repeat regions from the prn gene were previously made available or were acquired from all but one of the 122 isolates evaluated by MLST and ribotyping. Each isolate was assigned a repeat region allele based on the combined sequence of both regions. Thirty-four alleles were identified (Table 4). Although previously reported repeat region sequences derived from PCR amplicons for isolate 00-P-2730 comprise allele 1-4b1 / 2-6e1 (Diavatopoulos et al. 2005), allele 1-4b1 / 2-6a2 is found in 
genome sequence data for this isolate (Register et al. 2015b). Because other discrepant results have been reported by Diavatopoulos et al. (2005), as noted by Register et al. (2015a), here we opted to assign allele 1-4b1 / 2-6a2 to 00-P-2730. We did not further investigate the discrepancy, which could arise from either errors in data or reporting or from true sequence differences in cultures of 00-P-2730 maintained by different laboratories.

Only one allele, 1-4a2 / 2-6a1, appears to have a well-supported association with a particular host. Of 17 isolates with that allele all but one originated in seals on the Scottish, English or Danish coasts of the Atlantic Ocean and North Sea. These 16 isolates, which also share the same ST (ST11) and RT (RT19), were collected during a nearly 20-year span of time, from 1988 through 2006. The remaining isolate with a prn repeat region allele of 1-4a2 / 2-6a1 was cultured in 1996 from the pneumonic lung of a pig in South Dakota and otherwise types as ST6/RT2. Other ST6 or RT2 isolates from pigs were found among the group studied here (Tables 1 and 2), but none are both ST6 and RT2 (Table 3). The prn repeat region allele 1-4a3 / 2-5b1 was found in only two isolates, both from koalas. BLAST queries of Bordetella genome and nucleotide sequences currently available in GenBank did not identify any additional isolates having the same prn repeat region allele. A third koala isolate has allele 1-3a2 / 2-7a1, found more commonly and in a wide variety of mammalian hosts.

The only isolate for which prn repeat region sequences could not be acquired is 99-R0433, an RT30/ST60 isolate from a human in the United States. Repeated attempts to obtain PCR amplicons encompassing the repeat regions, using a variety of primers, were unsuccessful. Subsequent Southern blotting and restriction mapping suggested only a portion of the 3'end of the gene was likely to be present, with the GGXXP region and most or all of the PQP repeat region missing (K.B. Register, unpublished data). The genome sequence of this isolate was recently obtained (GenBank accession \#NZ_JGWN00000000) and provides further insight. 
The prn open reading frame is predicted to encode an 894 amino acid protein (versus 916 amino acids for the reference isolate RB50), but only $\sim 950 \mathrm{bp}$ at the $3^{\prime}$ terminus is recognizable as the pertactin gene. The 5 ' 1730 bp has only $33.1 \%$ nucleotide identity and $9.2 \%$ predicted amino acid identity with $p r n$ from RB50. In contrast, the 3'-terminal 950 bp has $92.9 \%$ nucleotide identity and $92.1 \%$ predicted amino acid identity with the collinear portion from RB50. Colinearity begins at the extreme 3' end of the PQP region and includes only a single predicted $\mathrm{PQP}$ repeat. Accordingly, it is not possible to classify isolate $99-\mathrm{R}-0433$ on the basis of prn repeat regions.

Pairing prn repeat region typing with MLST resulted in a total of 47 genotypes and an SID of 0.944 , as compared to 0.919 for MLST alone. Of the 13 STs with multiple members, 11 can be further delineated when prn repeat region alleles are considered (Table 4). Isolates with ST11 (16 seal isolates) and ST63 (2 koala isolates) could not be further differentiated.

$P v u I I$ ribotyping combined with prn repeat region typing yielded 54 genotypes and increased the SID from 0.920 to 0.954 . This approach resulted in further discrimination of isolates for all except 4 of the 16 RTs represented by more than a single isolate (Table 5). RTs that could not be subtyped when considering prn repeat region sequences include RT1 (2 isolates from pigs and 1 from a guinea pig), RT12 (2 rabbit isolates), RT19 (16 North Sea seal isolates) and RT22 (4 Caspian Sea seal isolates).

\section{Discussion}

Our analyses indicate that MLST and $P v u I I$ ribotyping are equally discriminatory methods for molecular typing of B. bronchiseptica. Because MLST offers many technical and practical advantages over ribotyping, we conclude that MLST is preferable as a primary typing tool. MLST data are objective, interpretation is straightforward and unambiguous and repeat analysis of isolates is rarely needed. In contrast, accurate assignment of ribotypes frequently 
requires repeated analysis due to slight run-to-run variability in restriction fragment patterns and the necessity of including appropriate reference standards on every gel. An additional limitation is that some isolates cannot be typed, as seen here. In contrast, MLST is highly standardized, scalable and portable and does not depend on comparisons with previously typed reference isolates. Comparisons of data acquired by different investigators and at different times are easily made, both within and between laboratories. A further benefit is the availability of a publically accessible, curated database serving as a central archive for typing data as well as for isolatespecific provenance and epidemiological information.

Although MLST alone is highly discriminatory, our results demonstrate that isolates sharing the same ST can frequently be further differentiated on the basis of prn repeat region sequences. This combined approach was informative for all STs represented by more than a single isolate except for ST11 $(n=16)$ and ST63 $(n=2)$. The ST63 isolates evaluated here were both cultured from koalas in Australia. As there is no additional information available about those isolates, we cannot determine whether there was a physical or epidemiological association between the animals of origin. Given the limited number available for our study, evaluation of additional, uniquely sourced ST63 isolates would better reveal the usefulness of prn repeat region sequencing for further distinguishing that ST. Considering the number and origin of ST11 isolates included in our study, it seems doubtful that the inability to subtype the group on the basis of prn repeat region sequences is due to insufficient sampling. These 16 isolates, which also share the same RT (RT19), were collected from seals during three successive outbreaks spanning nearly 20 years, from 1988 through 2006, and their geographic origin includes more than 15,000 miles of Atlantic Ocean and North Sea coastlines. Rather, the single prn repeat region allele they share is consistent with prior observations documenting a remarkable absence of genetic diversity among $B$. bronchiseptica isolates from seals as compared to isolates from other hosts (Register et al. 2015a). ST11/RT19 is the sole B. bronchiseptica genotype for which 
there is ample evidence of host restriction, having so far been found exclusively in isolates from either common seals (Phoca vitulina) or gray seals (Halichoerus grypus). The only additional ST represented here by more than a single isolate for which all isolates were acquired from the same host is ST63; both ST63 isolates are also RT6. However, the limited sample size for this ST prevents a sound determination as to whether it may be restricted to a single host.

Identification of an ST7 isolate from a third koala (Table 2) demonstrates that multiple genotypes of $B$. bronchiseptica are capable of infecting koalas.

Our discovery that B. bronchiseptica isolate $99-\mathrm{R}-0433$ has an aberrant prn gene lacking the repeat regions was unexpected. Approximately 10 years ago, investigators working with the closely related causative agent of whooping cough in humans, Bordetella pertussis, noted the emergence of strains that fail to express pertactin (Bouchez et al. 2009). Since that time, pertactin-deficient $B$. pertussis isolates have rapidly expanded worldwide, essentially replacing pertactin-producing strains in some countries, and the defects preventing expression of the gene have been characterized (Bouchez et al. 2009; Bowden et al. 2014; Pawloski et al. 2014; Williams et al. 2016). These include single nucleotide substitutions or insertions resulting in frameshift mutations and premature stop codons, the presence of an IS481 insertion sequence between the two repeat regions, promoter inversion and deletions of the promoter and/or a portion of the 5 ' end of the gene. In every case, both repeat regions are recognizable and intact. A few pertactin-deficient strains of B. bronchiseptica have also been identified, some dating as far back as the late 1980's (Register 2004; Diavatopoulos et al. 2005). All have one or two nucleotide insertions immediately prior to the GGXXP region, or just within it, that ultimately result in premature stop codons. Among the 122 isolates of B. bronchiseptica included in our study are seven that are predicted to be pertactin-deficient (pubMLST database ID numbers 77, 145, 453, 460, 466, 479 and 484); prn sequences for two of the seven have been reported previously (Register 2004; Diavatopoulos et al. 2005). Six of these isolates possess one of the 
same mutations already found in B. bronchiseptica while the seventh isolate, 99-R-0433, appears to be missing the majority of the gene, as noted above. Pertactin-deficient strains of $B$.

bronchiseptica appear to be rare. Collectively, we are aware of only 13 such isolates, 12 of which have intact repeat regions suitable for typing. The more extensive mutations seen recently in B. pertussis have not so far appeared in B. bronchiseptica and, with the exception of isolate 99-R-0433, all isolates for which prn sequences are available can be classified based on the two repeat regions as described here. Thus, it is expected that utilization of these repeat regions as a basis for subtyping will continue to be a reliable and informative adjunct approach, despite the remarkable degree of genetic variation associated with prn. The absence of one or both repeat regions could itself serve as a potentially discriminating feature, should such isolates continue to be only rarely found.

Considering the host of origin for isolates studied here, those from humans appear to possess the most genetic diversity, with representatives in 5/6 RT clusters and 5/7 ST clades, including those most divergent. Isolates from no other host are represented this extensively in either phylogeny. Although a larger number of isolates was evaluated from humans than from any other host, additional studies offer further evidence that the population of $B$. bronchiseptica infecting humans is highly diverse. Ribotyping data collected from prior publications (Register et al. 1997; Register and Magyar 1999; Register et al. 2000; Register 2004; Register et al. 2012; Register et al. 2015a) combined with data from this study reveal that $54.2 \%$ of human isolates $(n=25)$ comprise unique RTs, as compared to $21.4 \%$ from dogs $(n=28), 14.1 \%$ from rabbits $(\mathrm{n}=64), 5.1 \%$ from seals $(\mathrm{n}=59)$ and $3.5 \%$ from pigs $(\mathrm{n}=172)$. When MLST data from this study are considered together with results from previous reports (Buboltz 2008; Spilker et al. 2008) and unpublished data extracted from the Bordetella pubMLST database, $47.6 \%$ of human isolates $(n=85)$ possess unique STs, versus $45.4 \%$ from dogs $(n=22), 35.0 \%$ from rabbits $(n=28)$ and $26.1 \%$ from pigs $(n=23)$. At least some human infections are reasonably ascribed to 
nosocomial spread (Stevens-Krebbers et al. 1999; Huebner et al. 2006; Bose et al. 2008), but many others may arise from exposure to non-human sources since humans are not natural hosts for B. bronchiseptica (Goodnow 1980). Consequently, the diversity seen among human isolates may be a reflection of the wide host range the bacterium naturally infects. In some cases, typing a human isolate as described here may provide clues for pinpointing sources from which the bacterium was acquired. As an example, the human isolate St. Louis (pubMLST database ID 501) is an ST28/RT15 strain with a prn repeat region allele of 1-8a1 / 2-7d1. Five additional isolates in this study share that genotype, all from turkeys in California or in Midwestern states (pubMLST database ID numbers 489-492, 497). Isolate St. Louis was recovered from a 47-year old male with leukemia, residing in Missouri, whose condition rapidly deteriorated due to a respiratory infection. B. bronchiseptica was determined to be the causative agent and was recovered from sputum, bronchoscopy specimens and multiple blood cultures (J.P. Lang, personal communication). No additional details are available about this patient but an avian source of exposure seems plausible. A number of reports provide evidence to suggest that many human infections are the result of contact with infected or vaccinated pets (Gueirard et al. 1995; Dworkin et al. 1999; Foley et al. 2002; Berkelman 2003; Rath et al. 2008; Register et al. 2012; Brady et al. 2014; Garcia-de-la-Fuente et al. 2015). An example of typing data in support of this concept, acquired here and from a prior study (Register et al. 2012), is the finding that of $14 B$. bronchiseptica isolates known to be ST27/RT4, six were cultured from humans, seven from dogs or cats and one from a pig. Thirteen of the isolates share the prn repeat region allele 1-3a1 / 28a1; the remaining isolate has allele 1-3a1 / 2-9a1.

In conclusion, our data reveal MLST and $P v u I I$ ribotyping to be equally discriminatory methods for genotyping B. bronchiseptica and that subtyping on the basis of prn repeat region sequences permits higher order resolution of strains. While the application of all three approaches provides the maximum discriminatory power, MLST is the preferred primary typing 
method due to its objectivity, scalability, portability, and repeatability. MLST paired with prn repeat region typing was demonstrated here to be a highly discriminatory tool suitable for evaluating the population structure and investigating the epidemiology of B. bronchiseptica.

\section{Acknowledgments}

The authors gratefully acknowledge the excellent technical assistance of William Boatwright, Michael Mullins and Sarah Shore and thank Lea Ann Hobbs and David Alt at the NADC Genomics Unit for DNA sequence data. We are indebted to Geoff Foster, Ronald Welsh and Andy Tomlinson for sharing isolates from their private collections. This publication made use of the Bordetella Multilocus Sequence Typing website (http://pubmlst.org/ bordetella/) developed by Keith Jolley and sited at the University of Oxford (Jolley \& Maiden 2010, BMC Bioinformatics, 11:595). The development of this site has been funded by the Wellcome Trust. Mention of trade names or commercial products in this publication is solely for the purpose of providing specific information and does not imply recommendation or endorsement by the U.S. Department of Agriculture. USDA is an equal opportunity provider and employer.

\section{Conflict of Interest}

The authors declare that they have no conflict of interest.

\section{References}

Berkelman, R.L. (2003) Human illness associated with use of veterinary vaccines. Clin Infect Dis 37, 407-414. DOI: 10.1086/375595 
Bose, A., Javaid, W., Ashame, E., Kiska, D., Riddell, S. and Blair, D. (2008) Bordetella bronchiseptica: an emerging nosocomial pathogen in immunocompromised patients. Clin Microbiol Newsl 30, 117-119.

Bouchez, V., Brun, D., Cantinelli, T., Dore, G., Njamkepo, E. and Guiso, N. (2009) First report and detailed characterization of $B$. pertussis isolates not expressing pertussis toxin or pertactin. Vaccine 27, 6034-6041. DOI: 10.1016/j.vaccine.2009.07.074

Boursaux-Eude, C. and Guiso, N. (2000) Polymorphism of repeated regions of pertactin in Bordetella pertussis, Bordetella parapertussis, and Bordetella bronchiseptica. Infect Immun $\mathbf{6 8 ,}$ 4815-4817. DOI: 10.1128/IAI.68.8.4815-4817.2000

Bowden, K.E., Williams, M.M., Cassiday, P.K., Milton, A., Pawloski, L., Harrison, M., Martin, S.W., Meyer, S., Qin, X., DeBolt, C., Tasslimi, A., Syed, N., Sorrell, R., Tran, M., Hiatt, B. and Tondella, M.L. (2014) Molecular epidemiology of the pertussis epidemic in Washington State in 2012. J Clin Microbiol, 2, 3549-3557. DOI: 10.1128/JCM.01189-14

Brady, C., Ackerman, P., Johnson, M. and McNamara, J. (2014) Bordetella bronchiseptica in a pediatric cystic fibrosis center. J Cyst Fibros 13, 43-48. DOI: 10.1016/j.jcf.2013.08.002

Brockmeier, S.L., Register, K.B., Nicholson, T.L. and Loving, C.L. (2012) Bordetellosis. In Diseases of Swine ed. Zimmerman, J., Karriker, L., Ramirez, A., Schwartz, K. and Stevenson, G. pp. 670-679. Ames, IA: Wiley-Blackwell. 
Buboltz, A.M., Nicholson, T.L., Parette, M.R., Hester, S.E., Parkhill, J. and Harvill, E.T. (2008) Replacement of adenylate cyclase toxin in a lineage of Bordetella bronchiseptica. J Bacteriol 190, 5502-5511. DOI: 10.1128/JB.00226-08

Diavatopoulos, D.A., Cummings, C.A., Schouls, L.M., Brinig, M.M., Relman, D.A. and Mooi, F.R. (2005) Bordetella pertussis, the causative agent of whooping cough, evolved from a distinct, human-associated lineage of B. bronchiseptica. PLoS Pathog 1, e45. DOI: 10.1371/journal.ppat.0010045

Dworkin, M.S., Sullivan, P.S., Buskin, S.E., Harrington, R.D., Olliffe, J., MacArthur, R.D. and Lopez, C.E. (1999) Bordetella bronchiseptica infection in human immunodeficiency virusinfected patients. Clin Infect Dis 28, 1095-1099. DOI: 10.1086/514761

Foley, J.E., Rand, C., Bannasch, M.J., Norris, C.R. and Milan, J. (2002) Molecular epidemiology of feline bordetellosis in two animal shelters in California, USA. Prev Vet Med 54, 141-156. DOI: $10.1016 / \mathrm{S} 0167-5877(02) 00022-3$

Garcia-de-la-Fuente, C., Guzman, L., Cano, M.E., Aguero, J., Sanjuan, C., Rodriguez, C., Aguirre, A. and Martinez-Martinez, L. (2015) Microbiological and clinical aspects of respiratory infections associated with Bordetella bronchiseptica. Diagn Microbiol Infect Dis 82, 20-25. DOI: 10.1016/j.diagmicrobio.2015.01.011

Goodnow, R.A. (1980) Biology of Bordetella bronchiseptica. Microbiol Rev 44, 722-738. 
Gueirard, P., Weber, C., Le Coustumier, A. and Guiso, N. (1995) Human Bordetella bronchiseptica infection related to contact with infected animals: persistence of bacteria in host. J Clin Microbiol 33, 2002-2006.

Huebner, E.S., Christman, B., Dummer, S., Tang, Y.W. and Goodman, S. (2006) Hospitalacquired Bordetella bronchiseptica infection following hematopoietic stem cell transplantation. $J$ Clin Microbiol 44, 2581-2583. DOI: 10.1128/JCM.00510-06

Hunter, P.R. and Gaston, M.A. (1988) Numerical index of the discriminatory ability of typing systems: an application of Simpson's index of diversity. J Clin Microbiol 26, 2465-2466.

Jolley, K.A. and Maiden, M.C. (2010) BIGSdb: Scalable analysis of bacterial genome variation at the population level. BMC Bioinformatics 11, 595. DOI: 10.1186/1471-2105-11-595

Llombart, M., Chiner, E. and Senent, C. (2006) [Necrotizing pneumonia due to Bordetella bronchiseptica in an immunocompetent woman]. Arch Bronconeumol 42, 255-256.

Lo Re, V., 3rd, Brennan, P.J., Wadlin, J., Weaver, R. and Nachamkin, I. (2001) Infected branchial cleft cyst due to Bordetella bronchiseptica in an immunocompetent patient. J Clin Microbiol 39, 4210-4212.

Parkhill, J., Sebaihia, M., Preston, A., Murphy, L.D., Thomson, N., Harris, D.E., Holden, M.T., Churcher, C.M., Bentley, S.D., Mungall, K.L., Cerdeño-Tárraga, A.M., Temple, L., James, K., Harris, B., Quail, M.A., Achtman, M., Atkin, R., Baker, S., Basham, D., Bason, N., Cherevach, I., Chillingworth, T., Collins, M., Cronin, A., Davis, P., Doggett, J., Feltwell, T., Goble, A., 
Hamlin, N., Hauser, H., Holroyd, S., Jagels, K., Leather, S., Moule, S., Norberczak, H., O'Neil, S., Ormond, D., Price, C., Rabbinowitsch, E., Rutter, S., Sanders, M., Saunders, D., Seeger, K., Sharp, S., Simmonds, M., Skelton, J., Squares, R., Squares, S., Stevens, K., Unwin, L., Whitehead, S., Barrell, B.G. and Maskell, D.J. (2003) Comparative analysis of the genome sequences of Bordetella pertussis, Bordetella parapertussis and Bordetella bronchiseptica. Nat Genet 35, 32-40. DOI: 10.1038/ng1227

Pawloski, L.C., Queenan, A.M., Cassiday, P.K., Lynch, A.S., Harrison, M.J., Shang, W., Williams, M.M., Bowden, K.E., Burgos-Rivera, B., Qin, X., Messonnier, N. and Tondella, M.L. (2014) Prevalence and molecular characterization of pertactin-deficient Bordetella pertussis in the United States. Clin Vaccine Immunol 21, 119-125. DOI: 10.1128/CVI.00717-13

Rath, B.A., Register, K.B., Wall, J., Sokol, D.M. and Van Dyke, R.B. (2008) Persistent Bordetella bronchiseptica pneumonia in an immunocompetent infant and genetic comparison of clinical isolates with kennel cough vaccine strains. Clin Infect Dis 46, 905-908. DOI: $10.1086 / 528858$

Register, K.B. (2001) Novel genetic and phenotypic heterogeneity in Bordetella bronchiseptica pertactin. Infect Immun 69, 1917-1921. DOI: 10.1128/IAI.69.3.1917-1921.2001

Register, K.B. (2004) Comparative sequence analysis of Bordetella bronchiseptica pertactin gene (prn) repeat region variants in swine vaccines and field isolates. Vaccine 23, 48-57. DOI: 10.1016/j.vaccine.2004.07.020 
Register, K.B., Boisvert, A. and Ackermann, M.R. (1997) Use of ribotyping to distinguish Bordetella bronchiseptica isolates. Int J Syst Bacteriol 47, 678-683. DOI: 10.1099/00207713-47$3-678$

Register, K.B., Ivanov, Y.V., Harvill, E.T., Davison, N. and Foster, G. (2015a) Novel, hostrestricted genotypes of Bordetella bronchiseptica associated with phocine respiratory tract isolates. Microbiology 161, 580-592. DOI: 10.1099/mic.0.000035

Register, K.B., Ivanov, Y.V., Jacobs, N., Meyer, J.A., Goodfield, L.L., Muse, S.J., Smallridge, W.E., Brinkac, L., Kim, M., Sanka, R., Harvill, E.T. and Losada, L. (2015b) Draft genome sequences of 53 genetically distinct isolates of Bordetella bronchiseptica representing 11 terrestrial and aquatic hosts. Genome Announc 3, e00152-15. DOI: 10.1128/genomeA.00152-15

Register, K.B. and Magyar, T. (1999) Optimized ribotyping protocol applied to Hungarian Bordetella bronchiseptica isolates: identification of two novel ribotypes. Vet Microbiol 69, 277285. DOI: $10.1016 / \mathrm{S} 0378-1135(99) 00118-2$

Register, K.B., Sacco, R.E. and Foster, G. (2000) Ribotyping and restriction endonuclease analysis reveal a novel clone of Bordetella bronchiseptica in seals. J Vet Diagn Invest 12, 535540.

Register, K.B., Sukumar, N., Palavecino, E.L., Rubin, B.K. and Deora, R. (2012) Bordetella bronchiseptica in a paediatric cystic fibrosis patient: possible transmission from a household cat. Zoonoses Public Health 59, 246-250. DOI: 10.1111/j.1863-2378.2011.01446.x 
Sneath, P. and Sokal, R. (1973) The Principles and Practice of Numerical Classification. San Francisco: W.H. Freeman.

Spilker, T., Liwienski, A.A. and LiPuma, J.J. (2008) Identification of Bordetella spp. in respiratory specimens from individuals with cystic fibrosis. Clin Microbiol Infect 14, 504-506. DOI: 10.1111/j.1469-0691.2008.01968.x

Stevens-Krebbers, A.H., Schouten, M.A., Janssen, J. and Horrevorts, A.M. (1999) Nosocomial transmission of Bordetella bronchiseptica. J Hosp Infect 43, 323-324.

Tamura, K., Stecher, G., Peterson, D., Filipski, A. and Kumar, S. (2013) MEGA6: Molecular Evolutionary Genetics Analysis version 6.0. Mol Biol Evol 30, 2725-2729. DOI: 10.1093/molbev/mst197

Wernli, D., Emonet, S., Schrenzel, J. and Harbarth, S. (2011) Evaluation of eight cases of confirmed Bordetella bronchiseptica infection and colonization over a 15-year period. Clin Microbiol Infect 17, 201-203. DOI: 10.1111/j.1469-0691.2010.03258.x

Williams, M.M., Sen, K., Weigand, M.R., Skoff, T.H., Cunningham, V.A., Halse, T.A. and Tondella, M.L. (2016) Bordetella pertussis strain lacking pertactin and pertussis toxin. Emerg Infect Dis 22, 319-322. DOI: 10.3201/eid2202.151332

\section{Figure legends}


Fig. 1. Schematic representation of the PvuII ribotype patterns found among the 122 isolates evaluated in this study.

Fig. 2. Dendrogram and cluster analysis of PvuII B. bronchiseptica RTs performed according to UPGMA (tolerance, $1.0 \%$ ).

Fig. 3. UPGMA tree derived from MLST sequences of 122 B. bronchiseptica, isolates evaluated in this study. Branches corresponding to partitions reproduced in less than $50 \%$ of bootstrap replicates were collapsed. The percentages of replicate trees in which the associated taxa cluster together in the bootstrap test (1000 replicates) are indicated at each node. 
Table 1. Host distribution of B. bronchiseptica isolates among ribotypes (RT) and phylogenetically related RT clusters

\begin{tabular}{|c|c|c|c|c|c|c|c|c|c|c|c|c|c|c|}
\hline \multirow[b]{2}{*}{ Cluster } & \multirow[b]{2}{*}{ RT } & \multicolumn{12}{|c|}{ Host } & \multirow[b]{2}{*}{ Total } \\
\hline & & Human & Seal & Pig & Turkey & Rabbit & Dog & $\begin{array}{c}\text { Guinea } \\
\text { Pig }\end{array}$ & Horse & Cat & Koala & Leopard & Unknown & \\
\hline I & 2 & 0 & 0 & 4 & 0 & 0 & 0 & 0 & 0 & 1 & 0 & 0 & 0 & 5 \\
\hline I & 3 & 2 & 0 & 10 & 0 & 0 & 0 & 2 & 0 & 1 & 0 & 0 & 1 & 16 \\
\hline I & 4 & 7 & 0 & 1 & 0 & 1 & 8 & 0 & 2 & 3 & 0 & 0 & 0 & 22 \\
\hline I & 7 & 1 & 0 & 0 & 0 & 0 & 0 & 0 & 1 & 0 & 0 & 0 & 0 & 2 \\
\hline I & 10 & 1 & 0 & 0 & 0 & 0 & 0 & 0 & 0 & 1 & 0 & 0 & 0 & 2 \\
\hline I & 15 & 1 & 0 & 0 & 7 & 0 & 0 & 0 & 0 & 0 & 0 & 0 & 0 & 8 \\
\hline I & 17 & 0 & 0 & 0 & 0 & 1 & 0 & 0 & 0 & 0 & 0 & 0 & 0 & 1 \\
\hline I & 21 & 0 & 0 & 0 & 1 & 0 & 0 & 0 & 0 & 0 & 0 & 0 & 0 & 1 \\
\hline I & 23 & 0 & 0 & 1 & 0 & 0 & 0 & 0 & 0 & 0 & 0 & 0 & 0 & 1 \\
\hline I & 26 & 0 & 0 & 0 & 1 & 0 & 0 & 0 & 0 & 0 & 0 & 0 & 0 & 1 \\
\hline II & 1 & 0 & 0 & 0 & 0 & 1 & 0 & 2 & 0 & 0 & 0 & 0 & 0 & 3 \\
\hline II & 5 & 0 & 0 & 0 & 0 & 1 & 1 & 0 & 0 & 0 & 0 & 0 & 0 & 2 \\
\hline II & 8 & 0 & 0 & 0 & 0 & 0 & 0 & 0 & 2 & 0 & 0 & 0 & 0 & 2 \\
\hline II & 12 & 0 & 0 & 0 & 0 & 2 & 0 & 0 & 0 & 0 & 0 & 0 & 0 & 2 \\
\hline II & 19 & 0 & 16 & 0 & 0 & 0 & 0 & 0 & 0 & 0 & 0 & 0 & 0 & 16 \\
\hline II & 22 & 0 & 4 & 0 & 0 & 0 & 0 & 0 & 0 & 0 & 0 & 0 & 0 & 4 \\
\hline III & 6 & 0 & 0 & 0 & 0 & 0 & 0 & 0 & 0 & 0 & 3 & 1 & 0 & 4 \\
\hline III & 11 & 0 & 0 & 0 & 0 & 0 & 0 & 1 & 0 & 0 & 0 & 0 & 0 & 1 \\
\hline III & 13 & 0 & 0 & 0 & 0 & 0 & 1 & 0 & 0 & 0 & 0 & 0 & 0 & 1 \\
\hline III & 16 & 0 & 0 & 0 & 1 & 0 & 0 & 0 & 0 & 0 & 0 & 0 & 0 & 1 \\
\hline III & 27 & 1 & 0 & 0 & 0 & 0 & 0 & 0 & 0 & 0 & 0 & 0 & 0 & 1 \\
\hline III & 28 & 2 & 0 & 0 & 0 & 0 & 0 & 1 & 0 & 0 & 0 & 0 & 0 & 3 \\
\hline III & 31 & 1 & 0 & 0 & 0 & 0 & 0 & 0 & 0 & 0 & 0 & 0 & 0 & 1 \\
\hline IV & 14 & 1 & 0 & 0 & 0 & 0 & 0 & 0 & 0 & 0 & 0 & 0 & 0 & 1 \\
\hline IV & 18 & 2 & 0 & 1 & 1 & 0 & 0 & 1 & 0 & 0 & 0 & 0 & 0 & 5 \\
\hline IV & 20 & 0 & 0 & 0 & 1 & 0 & 0 & 0 & 0 & 0 & 0 & 0 & 0 & 1 \\
\hline IV & 24 & 0 & 0 & 0 & 0 & 0 & 0 & 0 & 1 & 0 & 0 & 0 & 0 & 1 \\
\hline $\mathrm{V}$ & 9 & 3 & 0 & 0 & 0 & 5 & 0 & 0 & 1 & 0 & 0 & 0 & 0 & 9 \\
\hline $\mathrm{V}$ & 25 & 0 & 0 & 0 & 0 & 1 & 0 & 0 & 0 & 0 & 0 & 0 & 0 & 1 \\
\hline $\mathrm{V}$ & 32 & 0 & 0 & 0 & 1 & 0 & 0 & 0 & 0 & 0 & 0 & 0 & 0 & 1 \\
\hline VI & 29 & 1 & 0 & 0 & 0 & 0 & 0 & 0 & 0 & 0 & 0 & 0 & 0 & 1 \\
\hline VI & 30 & 1 & 0 & 0 & 0 & 0 & 0 & 0 & 0 & 0 & 0 & 0 & 0 & 1 \\
\hline Total & & 24 & 20 & 17 & 13 & 12 & 10 & 7 & 7 & 6 & 3 & 1 & 1 & 121 \\
\hline
\end{tabular}


Table 2. Host distribution of $B$. bronchiseptica isolates among MLST sequence types (ST) and phylogenetically related ST clades

Host

\begin{tabular}{ccccccccccccccc} 
Clade & ST & Human & Seal & Pig & Turkey & Rabbit & Dog & Pig & Horse & Cat & Koala & Leopard & Unknown & Total \\
\hline I & 18 & 1 & 0 & 0 & 0 & 0 & 0 & 0 & 0 & 0 & 0 & 0 & 0 & 1 \\
I & 65 & 0 & 0 & 0 & 1 & 0 & 0 & 0 & 0 & 0 & 0 & 0 & 0 & 1 \\
I & 13 & 0 & 0 & 0 & 0 & 0 & 0 & 0 & 1 & 0 & 0 & 0 & 0 & 1 \\
I & 22 & 0 & 0 & 0 & 0 & 0 & 1 & 0 & 0 & 0 & 0 & 0 & 0 & 1 \\
I & 3 & 1 & 0 & 0 & 0 & 0 & 0 & 0 & 0 & 0 & 0 & 0 & 0 & 1 \\
I & 64 & 0 & 0 & 0 & 1 & 0 & 0 & 0 & 0 & 0 & 0 & 0 & 0 & 1 \\
I & 17 & 1 & 0 & 0 & 0 & 0 & 0 & 0 & 0 & 0 & 0 & 0 & 0 & 1 \\
I & 35 & 0 & 0 & 0 & 1 & 0 & 0 & 0 & 0 & 0 & 0 & 0 & 0 & 1 \\
I & 29 & 0 & 0 & 0 & 1 & 0 & 0 & 0 & 0 & 0 & 0 & 0 & 0 & 1 \\
I & 15 & 1 & 0 & 0 & 0 & 0 & 0 & 0 & 0 & 0 & 0 & 0 & 0 & 1 \\
I & 28 & 1 & 0 & 0 & 8 & 0 & 0 & 0 & 0 & 0 & 0 & 0 & 0 & 9 \\
I & 8 & 2 & 0 & 1 & 1 & 0 & 0 & 1 & 0 & 0 & 0 & 0 & 0 & 5 \\
I & 9 & 1 & 0 & 0 & 0 & 0 & 0 & 0 & 0 & 0 & 0 & 0 & 0 & 1 \\
I & 34 & 1 & 0 & 0 & 0 & 0 & 0 & 1 & 0 & 0 & 0 & 0 & 0 & 2 \\
II & 21 & 1 & 0 & 0 & 0 & 0 & 0 & 0 & 0 & 0 & 0 & 0 & 0 & 1 \\
III & 63 & 0 & 0 & 0 & 0 & 0 & 0 & 0 & 0 & 0 & 2 & 0 & 0 & 2 \\
IV & 62 & 0 & 0 & 0 & 0 & 0 & 0 & 0 & 0 & 1 & 0 & 0 & 0 & 1 \\
IV & 23 & 1 & 0 & 0 & 0 & 1 & 0 & 0 & 3 & 2 & 0 & 0 & 0 & 7 \\
IV & 33 & 0 & 4 & 0 & 0 & 0 & 1 & 0 & 1 & 0 & 0 & 1 & 0 & 7 \\
IV & 7 & 2 & 0 & 13 & 0 & 2 & 0 & 3 & 0 & 1 & 1 & 0 & 1 & 23 \\
IV & 12 & 3 & 0 & 0 & 0 & 3 & 0 & 0 & 0 & 0 & 0 & 0 & 0 & 6 \\
IV & 11 & 0 & 16 & 0 & 0 & 0 & 0 & 0 & 0 & 0 & 0 & 0 & 0 & 16 \\
IV & 6 & 0 & 0 & 2 & 0 & 2 & 0 & 0 & 2 & 0 & 0 & 0 & 0 & 6 \\
IV & 14 & 0 & 0 & 0 & 0 & 1 & 0 & 3 & 0 & 0 & 0 & 0 & 0 & 4 \\
V & 27 & 7 & 0 & 1 & 0 & 0 & 5 & 0 & 0 & 1 & 0 & 0 & 0 & 14 \\
V & 31 & 0 & 0 & 0 & 0 & 0 & 1 & 0 & 0 & 0 & 0 & 0 & 0 & 1 \\
VI & 10 & 0 & 0 & 0 & 0 & 3 & 1 & 0 & 0 & 0 & 0 & 0 & 0 & 4 \\
VI & 4 & 0 & 0 & 0 & 0 & 0 & 1 & 0 & 0 & 0 & 0 & 0 & 0 & 1 \\
VI & 5 & 0 & 0 & 0 & 0 & 0 & 0 & 0 & 0 & 1 & 0 & 0 & 0 & 1 \\
VII & 60 & 1 & 0 & 0 & 0 & 0 & 0 & 0 & 0 & 0 & 0 & 0 & 0 & 1 \\
\hline Total & & 24 & 20 & 17 & 13 & 12 & 10 & 8 & 7 & 6 & 3 & 1 & 1 & 122
\end{tabular}


Table 3. Distribution of B. bronchiseptica isolates among ribotypes (RT) versus MLST sequence types (ST)

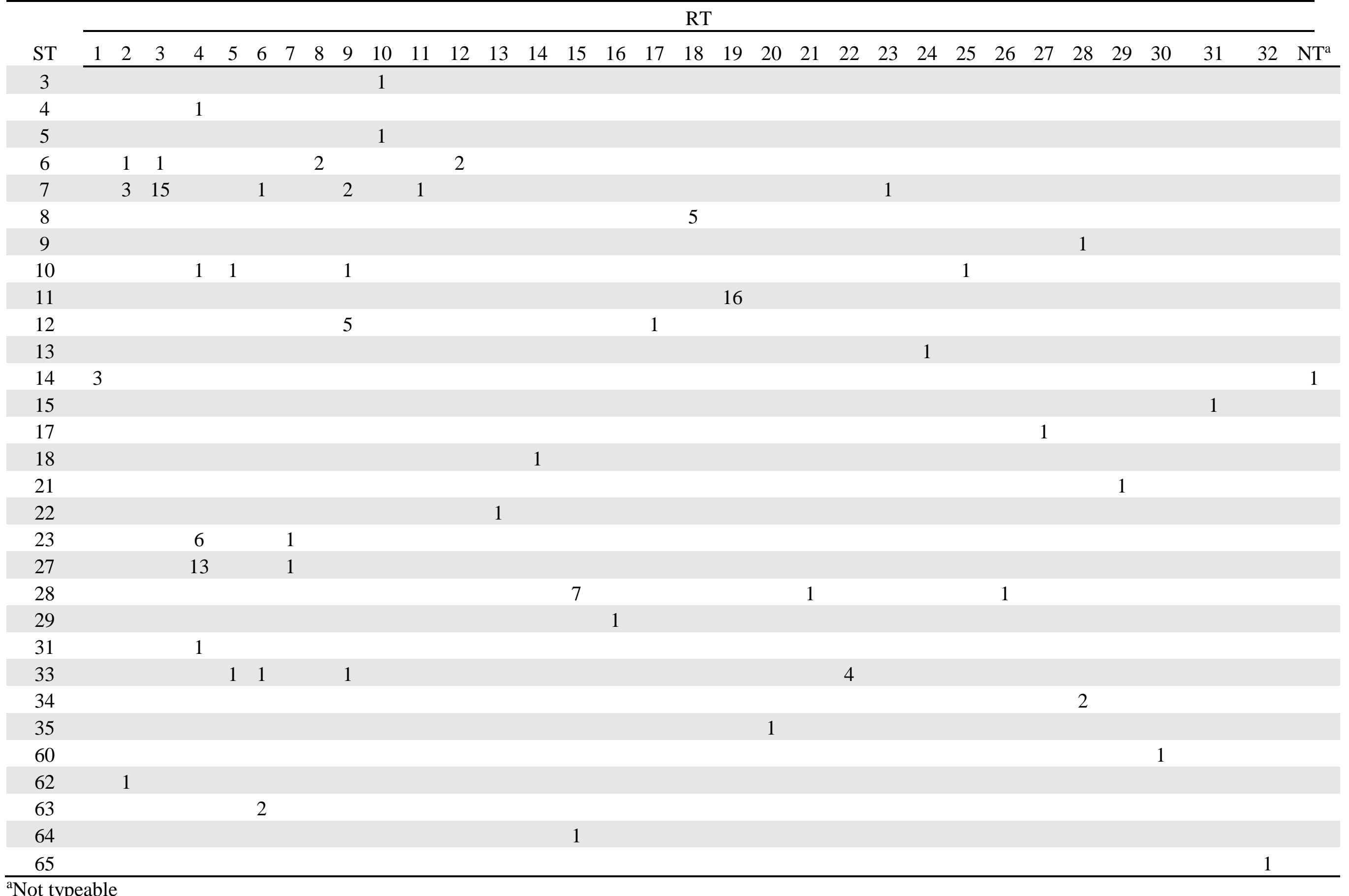


Table 4. Pertactin gene repeat region alleles and hosts of origin for MLST sequence types (ST) represented among the isolates evaluated

\begin{tabular}{|c|c|c|c|c|c|c|c|c|c|c|c|c|c|c|c|c|c|c|c|c|c|c|c|c|c|c|c|c|c|c|c|}
\hline \multirow[b]{2}{*}{ allele } & \multirow[b]{2}{*}{$\# \mathrm{Hbp}^{\mathrm{a}}$} & \multicolumn{29}{|c|}{ ST } & \multirow[b]{2}{*}{ Hosts of origin $(n)$} \\
\hline & & 3 & 4 & 5 & 6 & 7 & 8 & 9 & 10 & 11 & 12 & 13 & 14 & 15 & 17 & 18 & 21 & 22 & 23 & 27 & 28 & 29 & 31 & 33 & 34 & 35 & 62 & 63 & 64 & 65 & \\
\hline $1-3 \mathrm{a} 2 / 2-5 \mathrm{a} 1$ & 276 & & & & & 2 & & & & & & & & & & & & & & & & & & & & & & & & & $\operatorname{pig}(2)$ \\
\hline $1-3 a 2 / 2-6 b 1$ & 282 & & & & & & & & & & & & 1 & & & & & & & & & & & & & & & & & & guinea pig \\
\hline $1-3 \mathrm{a} 1 / 2-6 \mathrm{a} 1$ & 285 & & & & & & & & & & & & 3 & & & & & & & & & & & & & & & & & & guinea pig (2), rabbit \\
\hline $1-3 a 2 / 2-6 a 1$ & 285 & & & & & & & & & & & & & & & & & & & & & & & 1 & & & & & & & leopard \\
\hline $1-4 \mathrm{~b} 1 / 2-6 \mathrm{~d} 1$ & 285 & & & & & & & & & & & & & & & & & & & & & & & & & & & & & 1 & turkey \\
\hline $1-4 \mathrm{a} 3 / 2-5 \mathrm{~b} 1$ & 288 & & & & & & & & & & & & & & & & & & & & & & & & & & & 2 & & & koala (2) \\
\hline $1-4 \mathrm{~b} 1 / 2-6 \mathrm{e} 1$ & 291 & & & & & & & & & & & & & & & & & & & & 1 & & & & 1 & & & & & & turkey, guinea pig \\
\hline $1-3 \mathrm{a} 2 / 2-7 \mathrm{a} 1$ & 294 & & & & 1 & 17 & & & & & & & & & & & & & & & & & & 1 & & & & & & & $\begin{array}{l}\text { pig (7), guinea pig (3), rabbit (2), human (2), } \\
\text { dog, cat, horse, koala, unknown }\end{array}$ \\
\hline $1-3 \mathrm{a} 1 / 2-7 \mathrm{~b} 1$ & 297 & & & & & & & & & & & & & & & & & & 6 & & & & & & & & & & & & horse (2), cat (2), human, rabbit \\
\hline $1-4 \mathrm{~b} 1 / 2-7 \mathrm{e} 1$ & 297 & & & & & & & & & & & & & & & 1 & & & & & & & & & & & & & & & human \\
\hline $1-4 \mathrm{a} 1 / 2-6 \mathrm{a} 1$ & 300 & & & & 2 & & & & 3 & & 5 & & & & & & & & & & & & & 4 & & & & & & & rabbit (8), seal (4), human (2) \\
\hline $1-4 \mathrm{a} 2 / 2-6 \mathrm{a} 1$ & 300 & & & & 1 & & & & & 16 & & & & & & & & & & & & & & & & & & & & & seal (16), pig \\
\hline $1-4 \mathrm{~b} 1 / 2-6 \mathrm{a} 2$ & 300 & & & & & & & & & & & & & & & & & & & & & & & & 1 & & & & & & human \\
\hline $1-3 \mathrm{a} 2 / 2-8 \mathrm{e} 1$ & 303 & & & & & 4 & & & & & & & & & & & & & & & & & & & & & & & & & $\operatorname{pig}(4)$ \\
\hline $1-4 \mathrm{~b} 1 / 2-7 \mathrm{c} 1$ & 303 & & & & & & & & & & & & & & & & & 1 & & & & & & & & & & & & & $\operatorname{dog}$ \\
\hline $1-3 \mathrm{a} 1 / 2-8 \mathrm{a} 1$ & 306 & & 1 & & & & & & 1 & & & & & & & & & & & 13 & & & 1 & & & & & & & & $\operatorname{dog}(7)$, human (7), cat, pig \\
\hline $1-3 \mathrm{a} 2 / 2-8 \mathrm{a} 1$ & 306 & & & & 1 & & & & & & & & & & & & & & & & & & & & & & & & & & horse \\
\hline $1-5 \mathrm{e} 1 / 2-5 \mathrm{c} 1$ & 306 & & & & & & & & & & & & & 1 & & & & & & & & & & & & & & & & & human \\
\hline $1-3 \mathrm{a} 1 / 2-8 \mathrm{~g} 1$ & 309 & & & 1 & & & & & & & & & & & & & & & 1 & & & & & & & & & & & & horse, cat \\
\hline $1-4 \mathrm{a} 2 / 2-7 \mathrm{a} 1$ & 309 & & & & 1 & & & & & & & & & & & & & & & & & & & & & & & & & & pig \\
\hline $1-4 \mathrm{~b} 1 / 2-8 \mathrm{~b} 1$ & 309 & & & & & & & 1 & & & & & & & & & & & & & & & & & & & & & & & human \\
\hline $1-4 \mathrm{~b} 1 / 2-8 \mathrm{f} 1$ & 309 & 1 & & & & & & & & & & & & & & & & & & & & & & & & & & & & & human \\
\hline $1-5 \mathrm{~d} 1 / 2-6 \mathrm{a} 1$ & 315 & & & & & & & & & & 1 & & & & & & & & & & & & & & & & & & & & human \\
\hline $1-3 \mathrm{a} 1 / 2-9 \mathrm{a} 1$ & 318 & & & & & & & & & & & & & & & & & & & 1 & & & & & & & & & & & $\operatorname{dog}$ \\
\hline $1-5 f 1 / 2-6 f 1$ & 318 & & & & & & & & & & & & & & & & & & & & & & & & & & 1 & & & & cat \\
\hline $1-5 \mathrm{c} 1 / 2-7 \mathrm{f} 1$ & 318 & & & & & & & & & & & & & & & & 1 & & & & & & & & & & & & & & human \\
\hline $1-5 b 1 / 2-8 d 1$ & 318 & & & & & & 3 & & & & & & & & 1 & & & & & & & & & & & & & & & & pig, turkey, human, guinea pig \\
\hline $1-5 \mathrm{a} 1 / 2-8 \mathrm{c} 1$ & 321 & & & & & & & & & & & & & & & & & & & & & & & & & 1 & & & & & turkey \\
\hline $1-5 \mathrm{~b} 1 / 2-8 \mathrm{~b} 1$ & 324 & & & & & & 2 & & & & & & & & & & & & & & & & & & & & & & & & human (2) \\
\hline $1-4 a 4 / 2-9 b 1$ & 327 & & & & & & & & & & & & & & & & & & & & & & & 1 & & & & & & & horse \\
\hline $1-6 \mathrm{a} 1 / 2-6 \mathrm{c} 1$ & 342 & & & & & & & & & & & & & & & & & & & & & 1 & & & & & & & & & turkey \\
\hline $1-8 \mathrm{a} 1 / 2-6 \mathrm{~g} 1$ & 348 & & & & & & & & & & & & & & & & & & & & 1 & & & & & & & & & & turkey \\
\hline $1-8 \mathrm{a} 1 / 2-7 \mathrm{~d} 1$ & 357 & & & & & & & & & & & & & & & & & & & & 7 & & & & & & & & & & turkey (6), human \\
\hline $1-8 \mathrm{~b} 1 / 2-8 \mathrm{c} 1$ & 366 & & & & & & & & & & & 1 & & & & & & & & & & & & & & & & & 1 & & turkey, horse \\
\hline
\end{tabular}

${ }^{a}$ Combined length of GGXXP and PQP repeat regions 
Table 5. Pertactin gene repeat region alleles and hosts of origin for ribotypes (RT) represented among the isolates evaluated

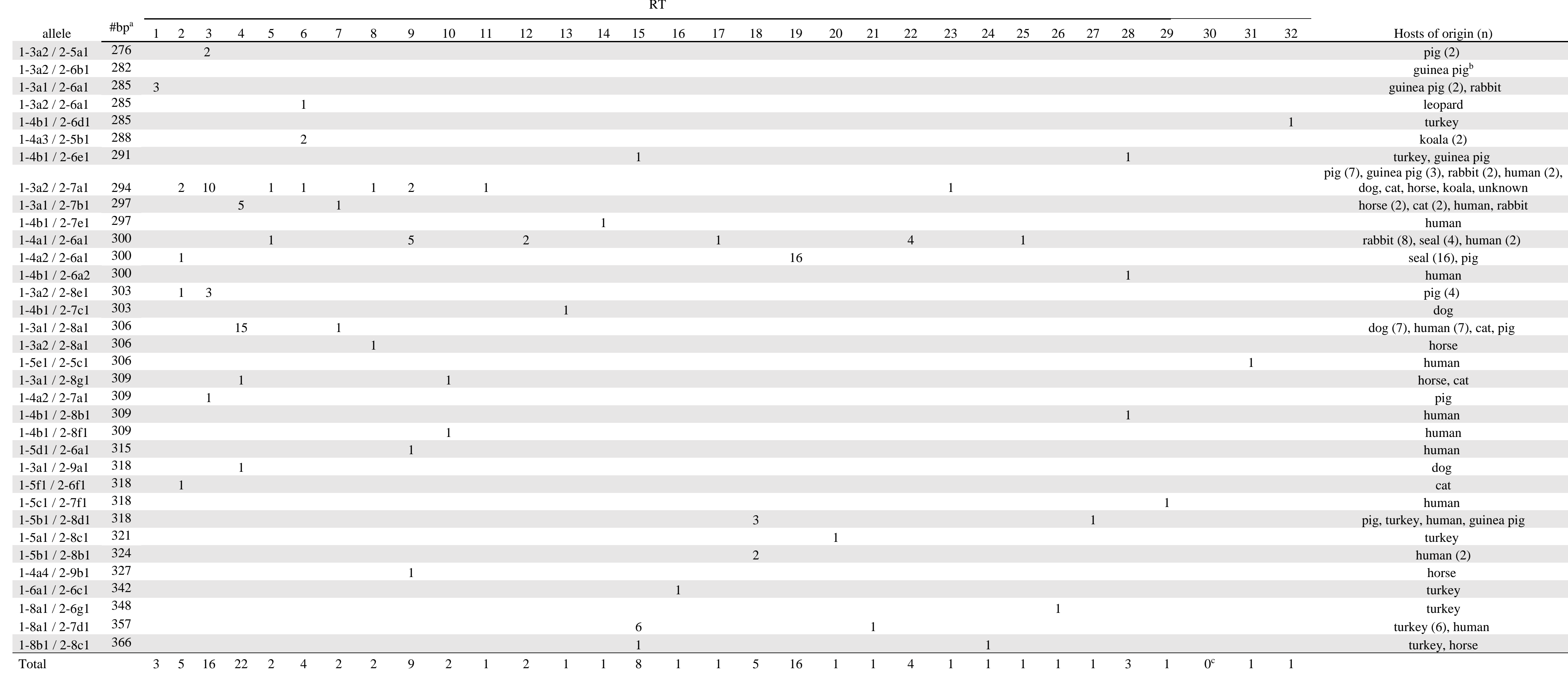

${ }^{\mathrm{a}}$ Combined length of GGXXP and PQP repeat regions

${ }^{\mathrm{b}}$ Not typeable

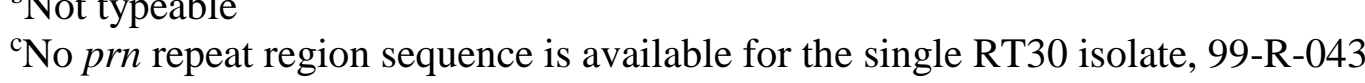




\section{Ribotype}

$\begin{array}{llllllllllllllllllllllllllllllll}1 & 2 & 3 & 4 & 5 & 6 & 7 & 8 & 9 & 10 & 11 & 12 & 13 & 14 & 15 & 16 & 17 & 18 & 19 & 20 & 21 & 22 & 23 & 24 & 25 & 26 & 27 & 28 & 29 & 30 & 31 & 32\end{array}$

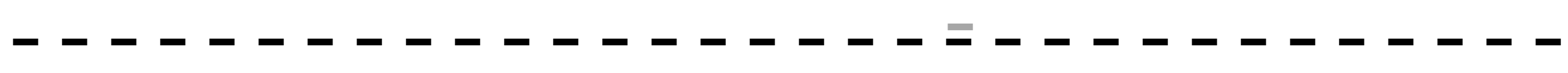
$-2$
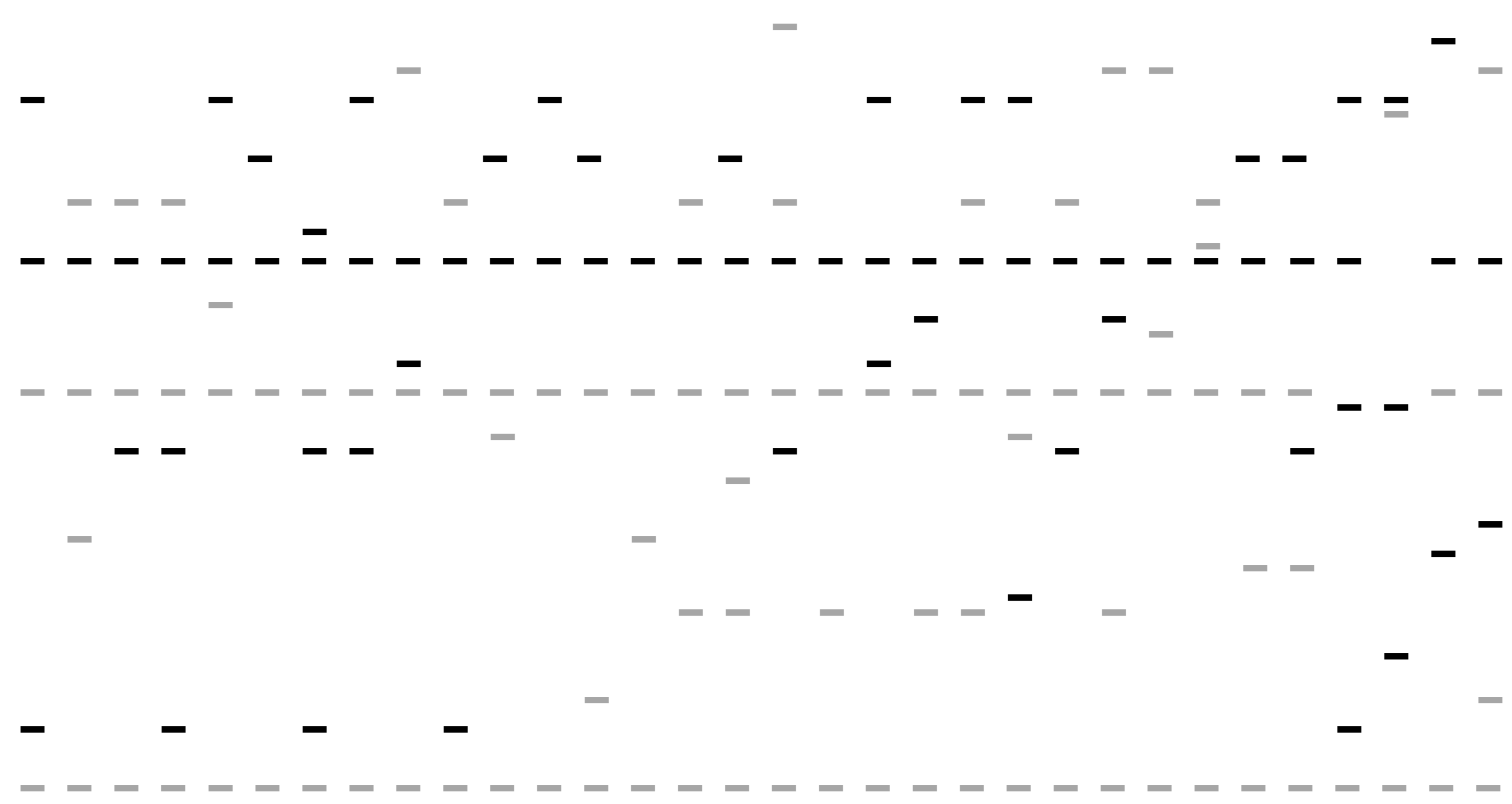

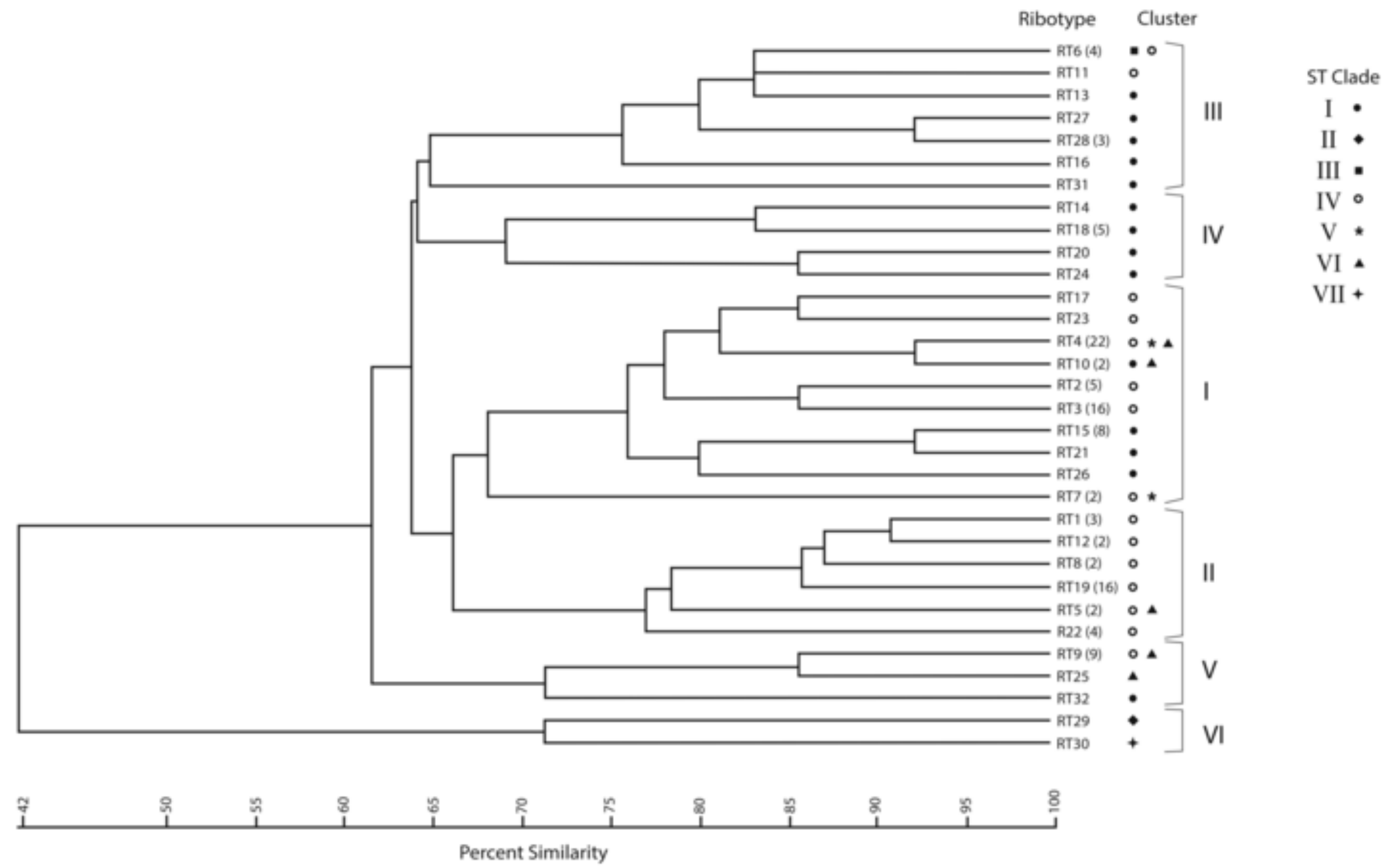
